\section{Descripción de los hallazgos en el perfil lipídico y proteico de pacientes dependientes a cocaína, en abstinencia reciente}

\author{
TERESA MASSARDO ${ }^{1}$, A. VERÓNICA ARAYA ${ }^{2}$, \\ CARLOS IBÁÑEZ ${ }^{3}$, JONATHAN VÉLIZ ${ }^{3}$, RENÉ FERNÁNDEZ ${ }^{1}$, \\ RODRIGO JAIMOVICH ${ }^{4}$, JULIO PALLAVICINI ${ }^{3}$, \\ ROLANDO CHANDÍA ${ }^{3}$, KARLA PEREIRA ${ }^{5}$, JAIME PEREIRA ${ }^{5}$
}

\section{Serum lipid levels in a group of cocaine dependent subjects in recent abstinence}

Background: Chronic cocaine users develop multiple potentially lethal ischemic vascular complications associated with accelerated atherosclerosis. Aim: To assess biochemical and lipid profiles among cocaine dependent subjects in recent abstinence. Material and Methods: A blood sample to measure blood count, biochemical and lipid profiles was obtained from 78 patients aged 19 to 53 years (78\% males) who complied with DSM-IV criteria for cocaine dependency. Laboratory results were compared with a group of normal subjects. Results: All cases had positive urinary cocaine, with a mean consumption lapse of 7.6 years. The frequency of smoking was higher in cases. Dependent males had higher body mass index than controls. Compared to controls, dependent females had significantly higher triglyceride (TG) levels and lower HDL cholesterol. Therefore the relation total/HDL cholesterol was higher $(p=0.0365)$. Dependent males had higher TG levels than their normal counterparts. Dependent subjects consuming cocaine base-paste had higher TG levels. Total proteins, albumin, urea and blood urea nitrogen were lower in dependent subjects. Among males, serum creatinine was lower and blood urea was positively correlated with the daily amount of cocaine use ( $p=0.03)$. After a month of strict abstinence, lipid profile was repeated in 27 patients and remained unchanged. Conclusions: Chronic cocaine use was associated with higher TG in both genders and lower HDL cholesterol in women when compared with a group of healthy counterparts.

(Rev Med Chile 2015; 143: 697-706)

Key words: Cocaine; Lipid profile; Protein metabolism.

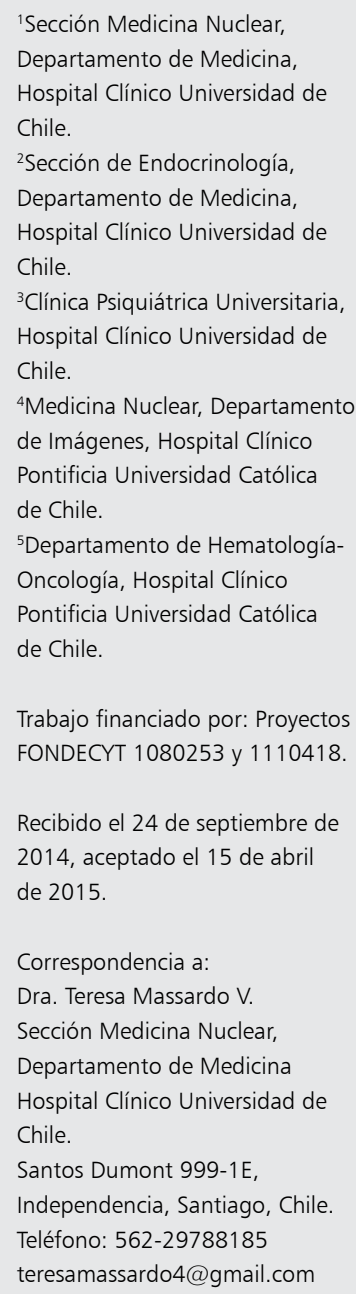

L a cocaína, metil $(1 R, 2 R, 3 S, 5 S)$-3-(benzoiloxi)-8-metil-8-azabiciclo [3.2.1] octane-2-carboxilato o benzoilmetilecgonina, alcaloide utilizado como droga de abuso, presenta importante proporción de población que se vuelve dependiente, especialmente, adultos jóvenes de sexo masculino. Se la considera la segunda droga más adictiva y la segunda más dañina, después de la heroína, entre las drogas recreacionales más populares. Este estimulante y anestésico local posee múltiples efectos adversos sobre el sistema nervioso central (SNC), árbol cardio y cerebrovascular, con eventos agudos coronarios e isquémicos cerebrales que pueden ser letales. Se ha observado también insuficiencia cardiaca secundaria y arritmias con coronarias 
normales. Otros parénquimas afectados son el pulmonar, renal, hepático, intestinal, muscular, piel, incluyendo mucosas oronasofaríngeas; además, se han descrito alteraciones sistémicas como fiebre y eosinofilia ${ }^{1-6}$. Su farmacología es compleja: vida media sanguínea aproximadamente $1 \mathrm{~h}$, con metabolismo sobre $90 \%$ hepático, excreción renal de ésteres benzoilecgonina y metilecgonina (que se pueden encontrar hasta $36 \mathrm{~h}$ post-administración). En el SNC, su efecto está mediado por bloqueo de reentrada presináptica de norepinefrina y dopamina, con exceso a nivel post-ganglionar. El consumo inhalatorio es el más común aunque por disponibilidad y costo, en nuestro medio se utiliza la pasta-base, producto intermedio de purificación que contiene cocaína como alcaloide principal.

Debido a sus propiedades simpaticomiméticas la cocaína induce vasoconstricción coronaria, taquicardia e hipertensión arterial. Las complicaciones isquémicas no son sólo explicables por vasoconstricción y parecen estar en relación a aterosclerosis acelerada y fenómenos de aterotrombosis. La muerte súbita asociada se ha descrito en $3,1 \%$ de autopsias consecutivas, encontrándose hipertrofia cardiaca, enfermedad obstructiva de pequeño vaso y enfermedad coronaria prematura con o sin trombosis luminal ${ }^{3}$. Las complicaciones médicas son heterogéneas ${ }^{7}$, sin embargo, el efecto sobre el metabolismo lipídico y proteico, así como sus perfiles después de consumo reciente son mayormente desconocidos. Esta información puede ser relevante considerando que el análisis de la placa lipídica en usuarios de cocaína ha revelado alta tasa de core lipídico a nivel carotídeo. Lo anterior plantea que el colesterol sanguíneo sería importante en el desarrollo de estas placas y que un manejo adecuado de la hipercolesterolemia ayudaría a prevenir eventos cerebrovasculares ${ }^{8}$. Por otra parte, la adicción a cocaína se caracteriza por deseo compulsivo de uso de la droga en detrimento de otras necesidades biológicas básicas, incluyendo un adecuado aporte nutricional, conducta que ha sido estudiada experimentalmente ${ }^{9}$.

\section{Objetivo}

El objetivo principal del estudio fue describir y analizar los hallazgos en alteraciones del perfil lipídico, bioquímico y hematológico en dependientes a cocaína de ambos sexos en consumo activo y después de un mes de abstinencia.

\section{Métodos}

Se estudiaron en forma prospectiva 78 pacientes (61 hombres), participantes de un proyecto de investigación entre 19 y 53 años de edad, con ingreso voluntario a unidad de adicciones para su desintoxicación. Para incluir los casos se requirió cumplir criterios DSM-IV (Diagnostic and Statistical Manual of Mental Disorders-IV version) de dependencia a cocaína como principal droga y presencia de metabolitos en orina por test rápido. Se excluyeron diabéticos, otras patologías psiquiátricas del eje I y enfermedades sistémicas crónicas conocidas (no hubo pacientes con daño hepático, nefropatía, o disfunción tiroidea), menores de 18 años y embarazo (mujeres en edad fértil tuvieron prueba sanguínea negativa). Los pacientes no estaban bajo efecto de hipolipemiantes orales.

Se efectuó al ingreso de la hospitalización un hemograma completo, velocidad de sedimentación eritrocitaria (VHS), creatininemia, perfil bioquímico con medición de transaminasa glutámico oxal acética (SGOT), láctico deshidrogenasa (LDH), fosfatasas alcalinas, bilirrubinemia, proteinemia, albuminemia, nitrógeno ureico (BUN), uremia, calcio y fósforo séricos; además, colesterol total, triglicéridos (TG), lipoproteína de alta densidad (HDL) y de baja densidad (LDL) calculada con fórmula de Friedewald $[\mathrm{LDL}=\mathrm{Co}-$ lesterol total+(HDL+TG/5) mg/dL $]^{10}$. Además se midió proteína $\mathrm{C}$ reactiva (PCR) ultrasensible.

Dentro de la primera semana del ingreso se efectuó análisis de cocaína y metabolitos en orina, con cromatografía de gas y espectrometría de masa.

Durante la desintoxicación estaba permitido el tabaco. La terapia incluía benzodiacepinas, haldol y risperidona, en dosis estándar, con el fin de disminuir la ansiedad inicial. Se consignó tiempo de consumo total de la droga (años) excluyendo abstinencias; se estimó la intensidad del consumo de cocaína (gramos/día) por los psiquiatras del grupo.

Los parámetros lipídicos estudiados al ingreso se repitieron al mes de abstinencia estricta controlada en subgrupo de 27 pacientes (6 mujeres). El resto de los parámetros se midieron a tiempos variables y no se incluyeron en el análisis.

Las variables se compararon con las de controles voluntarios del medio hospitalario, no consumidores de sustancias de abuso, salvo tabaco, que 
correspondían a 46 hombres y 15 mujeres, entre 19 y 58 años de edad.

Los valores de corte considerados anormales para lípidos fueron los siguientes: colesterol total $>200 \mathrm{mg} / \mathrm{dL}$, triglicéridos $>150 \mathrm{mg} / \mathrm{dL}, \mathrm{HDL}<40$ $\mathrm{mg} / \mathrm{dL}$ en hombres y $<45$ en mujeres, LDL $>100$ $\mathrm{mg} / \mathrm{dL}$ y la relación colesterol total/HDL $>4$ así como el colesterol no HDL $>150 \mathrm{mg} / \mathrm{dL}^{11}$.

El proyecto fue aprobado por los comités de ética de investigación de los centros participantes.

\section{Análisis de los resultados}

Las comparaciones de los promedios se realizaron mediante prueba de $t$ de student y Mann Whitney para datos no paramétricos, para variables categóricas se usó prueba de Fisher. Se efectuaron correlaciones de Pearson o Spearman según su distribución. La significación estadística fue $\mathrm{p}<0,05$. Además se realizó análisis bivariado y multivariado presentando Odds Ratio (OR) con sus respectivos Intervalos de Confianza [IC] de 95\%. Los valores de perfil lipídico que tuvieron seguimiento se compararon con prueba de Wilcoxon pareada.

\section{Resultados}

La Tabla 1 muestra los datos demográficos y de consumo y la Tabla 2 los parámetros bioquímicos, en ambos grupos. La principal droga

Tabla 1. Datos demográficos y de consumo de pacientes dependientes a cocaína y de controles

\begin{tabular}{|c|c|c|c|c|}
\hline \multirow[b]{2}{*}{ Datos demográficos } & \multicolumn{2}{|c|}{ Casos } & \multicolumn{2}{|c|}{ Controles } \\
\hline & $\begin{array}{c}\text { Hombres } \\
n: 61 \\
X \pm D E \\
\text { [rango] }\end{array}$ & $\begin{array}{c}\text { Mujeres } \\
n: 17 \\
X \pm D E \\
\text { [rango] }\end{array}$ & $\begin{array}{c}\text { Hombres } \\
n: 46 \\
X \pm D E \\
\text { [rango] }\end{array}$ & $\begin{array}{c}\text { Mujeres } \\
n: 15 \\
X \pm D E \\
\text { [rango] }\end{array}$ \\
\hline Edad (años) & $\begin{array}{c}31,8 \pm 8,2 \\
{[19-53]}\end{array}$ & $\begin{array}{c}37,1 \pm 8,5 \\
{[26-53]}\end{array}$ & $\begin{array}{c}31,6 \pm 8,7 \\
{[19-56]}\end{array}$ & $\begin{array}{c}38,7 \pm 9,1 \\
{[26-58]}\end{array}$ \\
\hline Masa $(\mathrm{kg})$ & $\begin{array}{c}81,2 \pm 12,6 \\
{[59-129]}\end{array}$ & $\begin{array}{c}63,2 \pm 6,8 \\
{[52-73]}\end{array}$ & $\begin{array}{c}79,5 \pm 9,5 \\
{[60-104]}\end{array}$ & $\begin{array}{c}68,8 \pm 8,9 \\
{[58-85]}\end{array}$ \\
\hline Talla (m) & $\begin{array}{l}1,73 \pm 0,06 \\
{[1,60-1,83]}\end{array}$ & $\begin{array}{l}1,61 \pm 0,08 \\
{[1,50-1,78]}\end{array}$ & $\begin{array}{l}1,75 \pm 0,05 \\
{[1,62-1,88]}\end{array}$ & $\begin{array}{l}1,63 \pm 0,06 \\
{[1,53-1,72]}\end{array}$ \\
\hline IMC $\left(\mathrm{kg} / \mathrm{m}^{2}\right)$ & $\begin{array}{c}28 \pm 4,8^{8} \\
{[22-48]}\end{array}$ & $\begin{array}{c}25,1 \pm 3,1 \\
{[20-30]}\end{array}$ & $\begin{array}{c}25,9 \pm 2,8^{8} \\
{[20-35]}\end{array}$ & $\begin{array}{c}26 \pm 3,9 \\
{[20-34]}\end{array}$ \\
\hline Escolaridad (años) & $\begin{array}{c}13,6 \pm 2,4 \\
{[8-20]}\end{array}$ & $\begin{array}{c}13,9 \pm 2,6 \\
{[10-19]}\end{array}$ & $\begin{array}{c}18,2 \pm 3,1 \\
{[12-23]}\end{array}$ & $\begin{array}{c}18,2 \pm 2,7 \\
{[14-23]}\end{array}$ \\
\hline \multicolumn{5}{|l|}{ Patrón de consumo } \\
\hline Edad de inicio cocaína (años) & $\begin{array}{c}21,4 \pm 7,8 \\
{[13-51]}\end{array}$ & $\begin{array}{c}24,5 \pm 1 \\
{[15-49]}\end{array}$ & & \\
\hline Tiempo de consumo cocaína (años) & $\begin{array}{c}7,8 \pm 4,9 \\
{[1-20]}\end{array}$ & $\begin{array}{c}7,6 \pm 5,7 \\
{[1-17]}\end{array}$ & & \\
\hline Intensidad consumo cocaína estimado (g/día) & $\begin{array}{l}1,8 \pm 1,4 \\
{[0,03-5,0]}\end{array}$ & $\begin{array}{l}1,78 \pm 1,6 \\
{[0,25-5,0]}\end{array}$ & & \\
\hline Producto consumo cocaína (g/día x año) & $\begin{array}{l}13,5 \pm 14 \\
{[0,16-65]}\end{array}$ & $\begin{array}{c}12,5 \pm 18,7 \\
{[0,86-68]}\end{array}$ & & \\
\hline Benzoilecgonina en orina $(\mathrm{ng} / \mathrm{mL})$ & $\begin{array}{c}797 \pm 2543 \\
{[0-17.258]}\end{array}$ & $\begin{array}{c}181 \pm 214 \\
{[21-591]}\end{array}$ & & \\
\hline Pasta-base de cocaína & $50 \%$ & $40 \%$ & & \\
\hline Alcohol & $95 \%$ & $80 \%$ & & \\
\hline Tetrahidrocanabinol & $66 \%$ & $43 \%$ & & \\
\hline Tabaquismo activo & $90 \%{ }^{\mathrm{f}}$ & $85 \% \S$ & $25 \%$ f & $33 \% \S$ \\
\hline
\end{tabular}

${ }^{s} p=0,014 ;{ }^{\&} p=0,039 ;{ }^{f} p<0,0001$. 
Tabla 2. Datos de pacientes dependientes a cocaína y de controles de hemograma, perfil bioquímico (las variables significativas descritas en Tabla 3)

\begin{tabular}{|lcccc|}
\hline Laboratorio & \multicolumn{2}{c}{ Casos } & \multicolumn{2}{c|}{ Controles } \\
& Hombres & Mujeres & Hombres & Mujeres \\
\hline Hematocrito (\%) & $45,8 \pm 2,7$ & $38 \pm 4,3$ & $45,5 \pm 2,4$ & $39,9 \pm 2,1$ \\
& {$[39,4-51,9]$} & {$[28,2-44,6]$} & {$[39,2-52,2]$} & {$[37-43,7]$} \\
\hline Hemoglobina (mg/dl) & $15,5 \pm 1,0$ & $12,6 \pm 1,9$ & $15,6 \pm 0,9$ & $13,4 \pm 0,8$ \\
& {$[13,2-17,8]$} & {$[8,2-15,1]$} & {$[13,3-17,3]$} & {$[12-14,7]$} \\
\hline Leucocitos (103/mm $)$ & $7,5 \pm 1,9$ & $7,1 \pm 1,2$ & $6,8 \pm 1,8$ & $6,7 \pm 1,7$ \\
& {$[3,8-12,4]$} & {$[4,6-9,4]$} & {$[4,1-14,2]$} & {$[4,2-11,8]$} \\
\hline Plaquetas (103/mm3) & $266 \pm 52,9$ & $295 \pm 99,9$ & $238 \pm 41,7$ & $277 \pm 66,7$ \\
& {$[112-396]$} & {$[188-564]$} & {$[165-373]$} & {$[169-397]$} \\
VHS (mm/hr) & $4,7 \pm 5,6$ & $8,2 \pm 5,5$ & $2,8 \pm 2,0$ & $5,7 \pm 4,3$ \\
& {$[1-28]$} & {$[1-22]$} & {$[1-9]$} & {$[1-14]$} \\
Creatininemia (mg/dl) & $0,89 \pm 0,1$ & $0,63 \pm 0,09$ & $0,99 \pm 0,1$ & $0,66 \pm 0,2$ \\
& {$[0,63-1,21]$} & {$[0,49-0,73]$} & {$[0,79-1,21]$} & {$[0,28-0,89]$} \\
\hline SGOT (U/L) & $34,1 \pm 37,7$ & $31,2 \pm 41,9$ & $30,2 \pm 24,4$ & $22,1 \pm 10,4$ \\
& {$[13-304]$} & {$[11-187]$} & {$[14-154]$} & {$[14-55]$} \\
Fosfatasas alcalinas (U/L) & $81,5 \pm 16,1$ & $71,4 \pm 20,6$ & $76 \pm 17,2$ & $71,1 \pm 19,2$ \\
& {$[44-127]$} & {$[42-117]$} & {$[36-122]$} & {$[41-112]$} \\
\hline Uremia (mg/dL) & $0,24 \pm 0,06$ & $0,22 \pm 0,08$ & $0,32 \pm 0,07$ & $0,31 \pm 0,07$ \\
& {$[0,10-0,41]$} & {$[0,11-0,24]$} & {$[0,21-0,50]$} & {$[0,22-0,42]$} \\
\hline Glicemia (mg/dL) & $86,9 \pm 17,7$ & $99,4 \pm 28,4$ & $83,8 \pm 8,6$ & $83,4 \pm 11,6$ \\
& {$[51-182]$} & {$[74-181]$} & {$[59-103]$} & {$[58-106]$} \\
\hline Ácido úrico (mg/dL) & $5,6 \pm 1,4$ & $3,6 \pm 1,0$ & $5,7 \pm 1,3$ & $3,9 \pm 0,8$ \\
& {$[2,3-9,8]$} & {$[2,1-5,9]$} & {$[2,5-9,8]$} & {$[2,5-5,5]$} \\
Calcio (mg/dL) & $9,5 \pm 0,4$ & $9,0 \pm 0,6$ & $9,6 \pm 0,3$ & $9,2 \pm 0,4$ \\
& {$[8,7-10,2]$} & {$[7,7-10,0]$} & {$[8,7-10,3]$} & {$[8,5-9,8]$} \\
\hline Fósforo (mg/dL) & $3,5 \pm 0,7$ & $3,6 \pm 0,8$ & $3,5 \pm 0,7$ & $3,4 \pm 0,5$ \\
& {$[2,1-5,1]$} & {$[2,0-4,8]$} & {$[2,6-5,8]$} & {$[2,4-4,2]$} \\
\hline
\end{tabular}

de dependencia fue clorhidrato de cocaína vía nasal; la pasta-base era utilizada en $48,7 \%$ como alternativa o en su ausencia; la mediana total de benzoilecgonina urinaria al ingreso fue 59,5 ng/ $\mathrm{mL}$, valor sin distribución normal con la prueba D'Agostino y Pearson $(\mathrm{p}<0,0001)$.

En ambos sexos, los TG estaban significativamente aumentados respecto a los controles ( $\mathrm{p}=0,0023$ en hombres y 0,0106 en mujeres); el colesterol LDL estaba disminuido $(\mathrm{p}=0,0197$ y 0,015 respectivamente); el colesterol no HDL [colesterol total-HDL] no tuvo diferencia significativa en ambos sexos ni en el grupo total $(\mathrm{p}=\mathrm{ns})$. Además, se comparó la relación Colesterol Total/ HDL y se observó que solamente en las mujeres dependientes fue más alto que en las controles $(\mathrm{p}=0,0365)$. En mujeres el colesterol HDL estaba disminuido ( $\mathrm{p}=0,0005)$. Las proteínas totales y la albuminemia también estaban disminuidas ( $p=0,0174 \mathrm{y}<0,0001$ respectivamente en hombres y $p=0,0252$ y 0,003 en mujeres), el BUN y la uremia estaban disminuidos $(\mathrm{p}<0,0001$ en hombres y $\mathrm{p}=0,0026$ y 0,0022 en mujeres). En hombres la creatininemia estaba disminuida $(\mathrm{p}=0,0006)$, sin valores sobre lo normal y las plaquetas aumentadas $(p=0,0035)$. Los hallazgos de los parámetros lipídicos se muestran en la Figura 1 y otros parámetros bioquímicos en la Figura 2.

En los dependientes de ambos sexos no hubo diferencias con los controles en los promedios de hematocrito, hemoglobina, leucocitos, glicemia, calcemia, fosfemia ni ácido úrico ni al comparar 


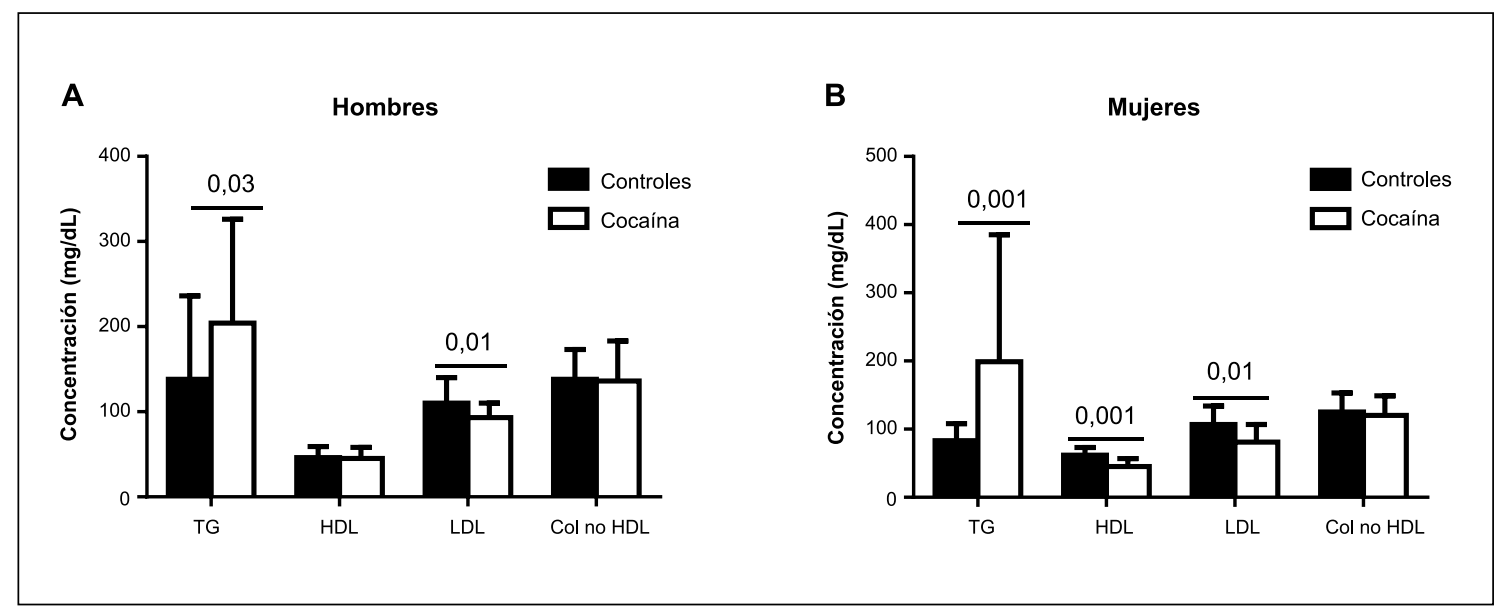

Figura 1. Valores promedio y desviación estándar de variables lipídicas en los dependientes a cocaína, en blanco, y en los controles, en negro, de sexo masculino (A) y femenino (B). TG: Triglicéridos; HDL: High Density Lipoprotein Cholesterol; LDL: Low Density Lipoprotein Cholesterol.
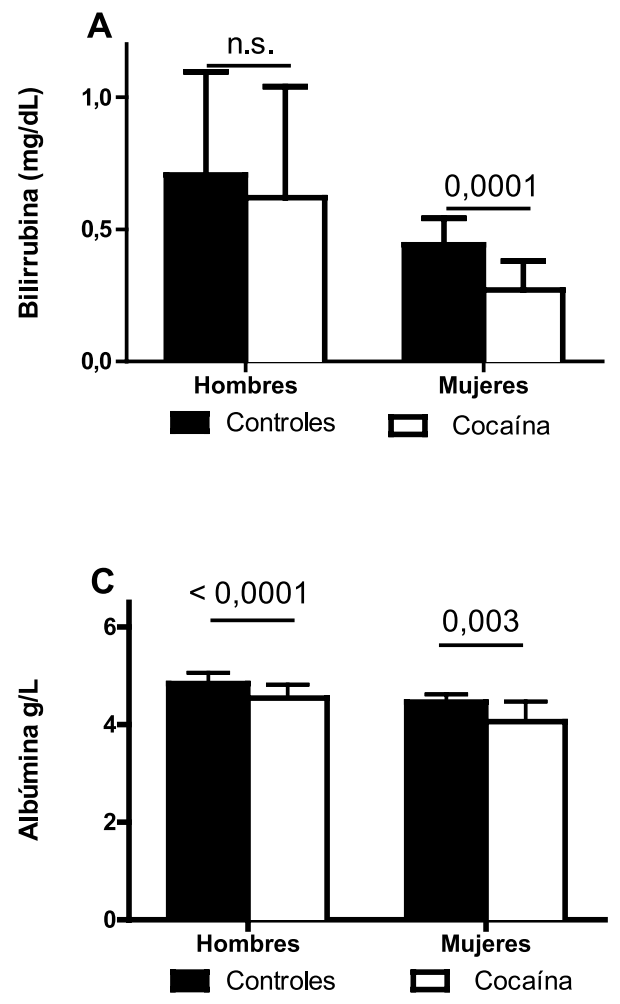
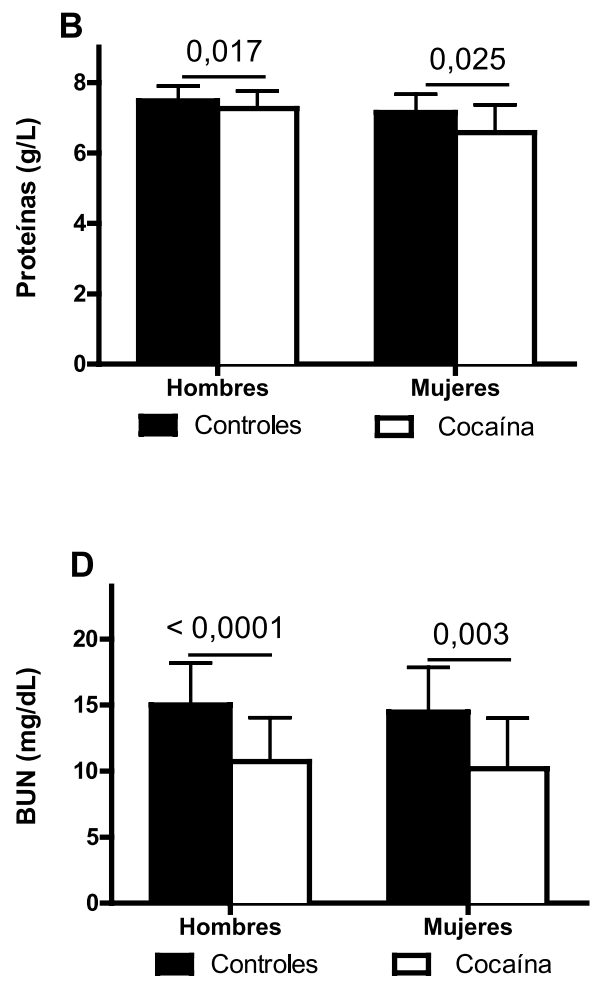

Figura 2. Valores promedio y desviación estándar de bilirrubinemia $(\mathbf{A})$, proteinemia (B), albúmina $(\mathbf{C})$ y nitrógeno ureico o BUN (D) en los dependientes a cocaína, en blanco, y en los controles, en negro. 
Tabla 3. Análisis bivariado y multivariado de parámetros bioquímicos plasmáticos con Odds Ratio (OR) e intervalos de confianza [IC] entre pacientes dependientes a cocaína y controles no consumidores de ambos sexos en conjunto

\begin{tabular}{|c|c|c|}
\hline Factor & OR [IC 95\%] & valor $p$ \\
\hline \multicolumn{3}{|c|}{ Análisis bivariado } \\
\hline Glucosa & $5,83[1,09-50,03]$ & 0,0198 \\
\hline Triglicéridos & $3,69[1,67-8,28]$ & 0,001 \\
\hline Colesterol LDL & $0,24[0,11-0,54]$ & $<0,001$ \\
\hline \multicolumn{3}{|c|}{ Análisis multivariado } \\
\hline Triglicéridos & $4,06[1,67-9,85]$ & 0,001 \\
\hline Colesterol LDL & $0,20[0,09-0,48]$ & $<0,001$ \\
\hline
\end{tabular}

promedios por sexo $(\mathrm{p}=\mathrm{ns})$; tampoco en SGOT, LDH ni fosfatasas alcalinas.

En mujeres dependientes, hubo hipoproteinemia en $41,1 \%$ vs $0 \%$ en controles ( $p=0,0077$ ); por otra parte, el promedio de albuminemia en los dependientes fue menor que en controles $(\mathrm{p}<0,0001)$. También hubo correlación inversa entre hemoglobina y plaquetas circulantes en los casos de mujeres dependientes $(r=-0,63$; $\mathrm{p}=0,008)$ no observada en los otros grupos.

Los parámetros significativos para Odds Ratio se muestran en la Tabla 3; en análisis bivariado se observó que los dependientes a cocaína tenían más alta probabilidad de presentar niveles alterados de glicemia, triglicéridos y colesterol LDL que los controles sin cocaína; en el análisis multivariado, era significativo para los dependientes, la probabilidad más alta de presentar niveles alterados de triglicéridos y colesterol LDL. La relación colesterol total/HDL ni el colesterol no HDL tuvieron significancia entre los grupos con ninguno de esos análisis $(\mathrm{p}=\mathrm{ns})$.

Al comparar los dependientes a cocaína usuarios o no de pasta-base de ambos sexos, se demostró que el promedio de los TG fue mayor en los usuarios de pasta-base $(p=0,0061)$, Figura 3 A. El promedio de ácido úrico fue también mayor en los consumidores de pasta-base $(\mathrm{p}=0,0489)$ así como el de bilirrubinemia $(\mathrm{p}=0,0122)$, más marcado en hombres $(p=0,0038)$.

Además, se observó que al comparar dependientes a cocaína que utilizaban alcohol con aquellos que no lo consumían, el promedio de urea fue mayor en los primeros $(\mathrm{p}=0,0134)$ así como el de ácido úrico $(\mathrm{p}=0,0297)$ y BUN $(\mathrm{p}=0,026)$, diferencia que fue más significativa en mujeres $(\mathrm{p}=0,0105)$.

No hubo diferencias en la PCR ultrasensible entre pacientes y controles, $6,85 \pm 4,6 \mathrm{mg} / \mathrm{L}$ y 4,9 $\pm 4,0 \mathrm{mg} / \mathrm{L}$ respectivamente $(\mathrm{p}=\mathrm{ns})$.

En el grupo de dependientes, el impacto de variables de dependencia a cocaína sobre los parámetros plasmáticos estudiados mostró correlación positiva entre intensidad de consumo, expresado como gramo/día de cocaína con uremia $(r=0,2537 ; p=0,03)$ como se muestra en la Figura $3 \mathrm{~B}$. En el grupo de hombres dependientes, hubo correlación inversa entre años de consumo de droga con HDL ( $r=-0,227 ; p=0,033)$ (Figura $3 \mathrm{C}$ ).

En el sub-análisis de seguimiento, luego de un mes de abstinencia controlada, no se observaron cambios significativos en los valores de lípidos ni específicamente de TG ( $\mathrm{p}=\mathrm{ns})$; este grupo recibió alimentación con régimen común en hospitalización. Tampoco hubo diferencia en la relación colesterol total/HDL ni en el colesterol no HDL $(\mathrm{p}=\mathrm{ns})$.

\section{Discusión}

Los usuarios crónicos de cocaína tienen alteraciones cerebrales que podrían estar relacionadas con variaciones del comportamiento, que finalmente inciden en su entorno ${ }^{12}$. En un meta-análisis sobre efectos neurocognitivos a largo plazo, se describe un amplio rango de funciones deterioradas, planteándose como fenómeno subyacente el desarrollo de ateroesclerosis asociado al uso de la droga ${ }^{13}$. En dependientes, hemos observado alteraciones de perfusión parenquimatosa cerebral asociadas a conductas de tipo perseverativo. Por otra parte, existe evidencia de disfunción endotelial importante ${ }^{14}$, así como marcadores de daño vascular y activación de plaquetas circulantes ${ }^{15}$, fenómenos que podrían jugar un papel fisiopatológico en las alteraciones cerebrales asociadas al consumo ${ }^{16}$. Nuestros casos tenían aumento de plaquetas circulantes, hallazgo para lo cual no tenemos explicación clara pero que pensamos no tiene significancia biológica debido al amplio rango de normalidad en esta línea celular.

La observación más significativa del presente trabajo es el aumento de TG en dependientes a cocaína de ambos sexos y su asociación con el consumo de pasta-base, lo cual no había sido reportado, Figuras 1 y 3 A. Modelos experimentales 
A

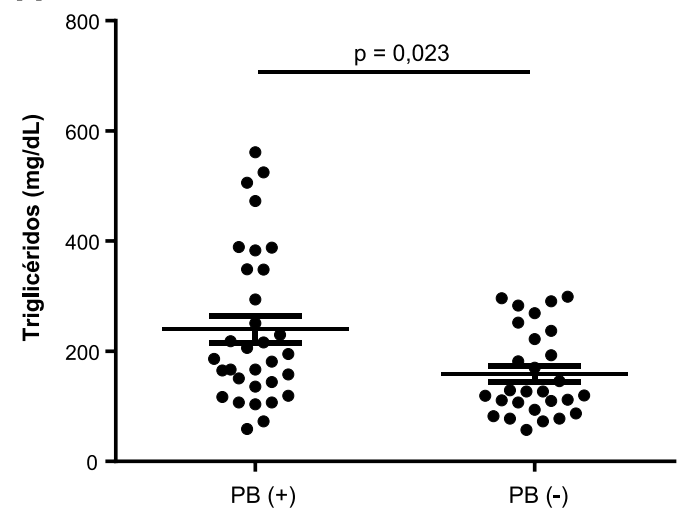

B

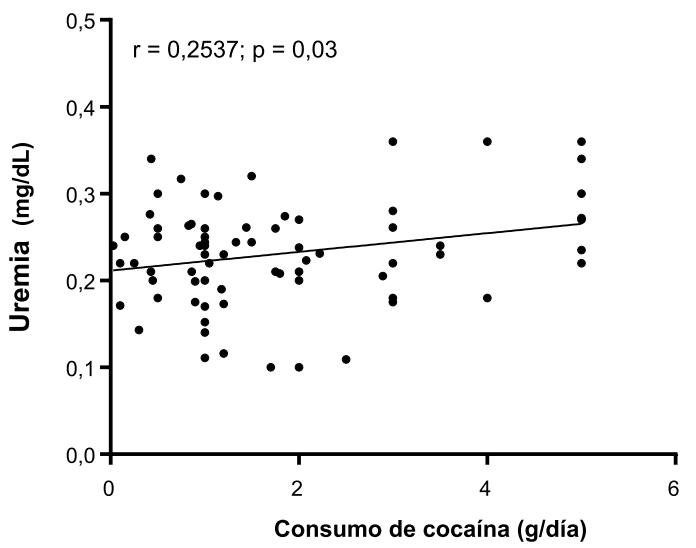

C

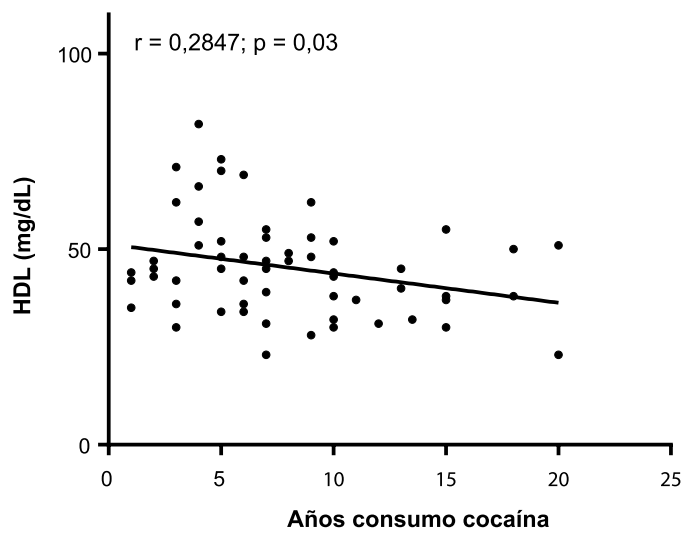

Figura 3. Distribución de valores de triglicéridos en dependientes a cocaína con consumo adicional de pasta-base, a izquierda y sin este, a la derecha (A). Correlación entre colesterol HDL y longitud de consumo de cocaína en los dependientes de sexo masculino (B). Correlación entre uremia e intensidad de consumo de cocaína estimado en dependientes de ambos sexos (C). TG: Triglicéridos; HDL: High Density Lipoprotein Cholesterol; Col T/HDL: Colesterol Total/HDL.

han demostrado que la cocaína tiene efectos sobre el metabolismo lipídico hepático, aumentando la salida de TG a la circulación, la peroxidación de lípidos y alteraciones del metabolismo del colesterol, lo que puede ser más marcado si hay malnutrición proteica ${ }^{17,18}$. Esto podría ser perpetuado por el uso crónico de cocaína, lo que explicaría que abstinencia estricta corta no logró revertir las alteraciones del perfil lipídico en el subgrupo estudiado.

Por otra parte, es habitual que los dependientes a cocaína sean consumidores de alcohol. En este grupo, 90\% lo consumía habitualmente en con- junto con cocaína, lo cual podría explicar también el aumento de los TG.

El menor colesterol-LDL observado en dependientes podría atribuirse a disminución de síntesis hepática de lipoproteína de muy baja densidad o VLDL, por daño asociado a uso de cocaína, alcohol y/o malnutrición, sin embargo, como no fue posible realizar medición directa del LDL, se debe considerar la limitación propia de la fórmula utilizada, en TG sobre $400 \mathrm{mg} / \mathrm{dL}$, lo que pudo influir en el promedio. Por otra parte, al comparar el colesterol no HDL, no encontramos una diferencia significativa. 
El colesterol-HDL estuvo significativamente disminuido en mujeres dependientes y también la relación colesterol total/HDL aumentada, no así en hombres. Está descrito que en la hepatotoxicidad por cocaína, la peroxidación de lípidos inducida por estrés oxidativo se encuentra relacionada con las hormonas sexuales, especialmente la testosterona, con menor daño en mujeres con ovarios intactos ${ }^{19,20}$. Sin embargo, nuestros hallazgos se contraponen a estas observaciones, probablemente debido al menor número de mujeres, por lo que no podemos aseverar que esté relacionado con el efecto de hormonas sexuales como ha sido descrito para otras alteraciones.

Parece existir un efecto modulatorio de la progesterona sobre la cocaína en relación a los efectos del comportamiento en distintas especies. Hay diferencias en activación neural y en respuestas al craving y al estrés entre géneros, no bien establecidas, que no parecen asociarse con la farmacocinética de la droga que podrían estar atenuados por la progesterona ${ }^{21,22}$.

En nuestros pacientes masculinos en abstinencia reciente, se pesquisó IMC levemente mayor respecto a los controles, lo que contrasta con estudio de dependientes de cocaína, en quienes no se encontró disminución significativa de IMC pero si un menor peso, sugerente de que el uso crónico interfiere con procesos metabólicos y determinaría desbalance entre ingesta y almacenamiento de grasa ${ }^{23}$.

El neuropéptido CART (cocaine-amphetamine regulated transcript) tiene efecto regulatorio de la ingesta alimentaria, cuya expresión está agudamente aumentada post-administración de cocaína ${ }^{24,25}$; que podría explicar una excesiva ganancia de peso al discontinuar consumo. Por otra parte, es conocido que el uso de marihuana provoca cambios en la ingesta dietética, con mayor consumo de calorías, proteínas y grasas, sin embargo, se ha observado que no produce cambios en el peso $^{26}$. Casi dos tercios de nuestros pacientes consumían marihuana en forma habitual y aunque no hubo información dietética pre-hospitalización, ellos tenían peso similar a los controles.

Una explicación para los mayores niveles de glicemia en algunos dependientes, si bien sin significancia estística, es que la cocaína a través de acción adrenérgica, puede determinar una disminución de la liberación de insulina y aumento de glucagón, con la consecuente alza de la glicemia ${ }^{27}$.
Lamentablemente, en este estudio no fue posible evaluar niveles de insulina ni péptido $C$ que habrían permitido confirmar esta hipótesis. También pudiera ser que, a pesar de la indicación de ayuno, por las características sicológicas propias de estos pacientes, no lo hubiesen cumplido.

Otro hallazgo fue el menor promedio de creatinina y BUN plasmáticos en mujeres, que podría traducir disminución de masa muscular y dieta carente de nutrientes proteicos, lo que se refleja en el menor promedio de proteínas y albúmina plasmática en ambos sexos. Si a esto se suma una probable falta de ejercicio que se pudiera ver reflejada en HDL bajo, podría especularse que los dependientes tienen sarcopenia, que favorecería trastornos metabólicos como insulino-resistencia y dislipidemia, asociados a mayor riesgo cardiovascular.

Los individuos dependientes a cocaína tienen un estado inflamatorio basal elevado. Drogas que producen adicción presentarían efectos inmunomodulatorios con alteraciones de interleukina 6 (IL-6) en saliva y elevación de factor de necrosis tumoral (TNF-alfa) pro-inflamatorio, frente a estrés por estímulo con imágenes de disgusto ${ }^{28,29}$. Las citoquinas pro-inflamatorias, marcadores de riesgo metabólico y de aterosclerosis, también favorecerían la mayor frecuencia de estos problemas en adictos. Sin embargo, en nuestros casos no hubo diferencia significativa en el nivel de PCR ultrasensible, que podría ser explicado porque los controles eran fumadores.

Existe evidencia de que alteraciones en los lípidos sanguíneos pueden afectar la función de las plaquetas; de hecho, se ha demostrado que tanto la hipertrigliceridemia familiar ${ }^{30,31}$ como aguda $^{32}$, se asocia a hiperactividad plaquetaria. En este sentido, en usuarios crónicos de cocaína el aumento observado del nivel de TG podría jugar algún papel en la activación de las plaquetas in vivo ${ }^{15}$. El aumento significativo del recuento plaquetario en mujeres se correlacionó con menor nivel de hemoglobina, lo que probablemente se explique por deficiencia de hierro.

Por otra parte, se ha descrito, recientemente, déficit de la capacidad antioxidante total en dependientes a estimulantes, incluyendo cocaína, que pudiera estar involucrada en daño parenquimatoso del cerebro y otros órganos ${ }^{33}$.

La correlación positiva entre tiempo de consumo de cocaína y disminución de HDL en hombres, es un hallazgo interesante en el sentido de consi- 
derar otro factor de riesgo que podría contribuir al desarrollo de aterosclerosis acelerada asociada al uso crónico de la droga.

\section{Limitaciones $y$ fortalezas}

Pensamos que su principal fortaleza es ser un estudio prospectivo, de un número relativamente importante de pacientes con consumo comprobado reciente de cocaína, en hospitalización voluntaria para abstinencia controlada. Hubiera sido interesante aumentar el número de mujeres y prolongar la abstinencia, dificultosa en este tipo de dependientes y haber considerado medir niveles de insulinemia para cálculo de índice HOMA, lo cual se podría efectuar en otro estudio posterior. Como se mencionó, lamentablemente, en este estudio no fue posible evaluar niveles de insulina ni péptido C. Tampoco contamos con medición de parámetros específicos de inflamación o de componente graso como citoquinas o leptinas ni tampoco datos exactos en pacientes ni controles de actividad física, ni dieta que son difíciles de estimar en sujetos con consumo activo reciente de sustancias?.

\section{Conclusión}

El consumo crónico de cocaína en pacientes con criterios de dependencia se asoció a disminución de proteínas plasmáticas y alteraciones lipídicas, caracterizadas por aumento de TG en ambos sexos y disminución de HDL así como aumento de la relación colesterol total/HDL en mujeres, comparando con controles. Esto podría contribuir a la génesis de ateroesclerosis acelerada. Estas alteraciones se explicarían por cambios dietarios, actividad física, consumo de alcohol y efecto de la droga sobre el metabolismo de proteínas u otros y debieran considerarse en el manejo terapéutico global del paciente.

Agradecimientos: Al Sr. Ariel Castro Q.F. de la oficina de apoyo a la investigación clínica (OAIC) del Hospital Clínico Universidad de Chile por su rigurosa colaboración en el análisis estadístico.

\section{Referencias}

1. Nutt D, King LA, Saulsbury W, Blakemore C. Development of a rational scale to assess the harm of drugs of potential misuse. Lancet 2007; 369 (9566): 1047-53.

2. De Giorgi A, Fabbian F, Pala M, Bonetti F, Babini I, Bagnaresi I, et al. Cocaine and acute vascular diseases. Curr Drug Abuse Rev 2012; 5 (2): 129-34.

3. Lucena J, Blanco M, Jurado C, Rico A, Salguero M, Vázquez R, et al. Cocaine-related sudden death: a prospective investigation in south-west Spain. Eur Heart J 2010; 31 (3): 318-29.

4. Massardo T, Pino A, Berrocal I, Castro G, Prat H, Pereira J. [Cocaine-induced vascular damage. Report of one case]. Rev Med Chile 2012; 140 (4): 507-11.

5. Restrepo CS, Carrillo JA, Martínez S, Ojeda P, Rivera AL, Hatta A. Pulmonary complications from cocaine and cocaine-based substances: imaging manifestations. Radiographics 2007; 27 (4): 941-56.

6. Hoefsloot W, de Vries RA, Bruijnen R, Bosch FH. Renal infarction after cocaine abuse: a case report and review. Clin Nephrol 2009; 72 (3): 234-6.

7. Pavarin R, Lugoboni F, Mathewson S, Ferrari AM, Guizzardi G, Quaglio G. Cocaine-related medical and trauma problems: a consecutive series of 743 patients from a multicentre study in Italy. Eur J Emerg Med 2011; 18 (4): 208-14.

8. Du J, Wasserman BA, Tong W, Chen S, Lai S, Malhotra $\mathrm{S}$, et al. Cholesterol is associated with the presence of a lipid core in carotid plaque of asymptomatic, young-tomiddle-aged African Americans with and without HIV infection and cocaine use residing in inner-city Baltimore, Md., USA. Cerebrovasc Dis 2012; 33 (3): 295-301.

9. Perry AN, Westenbroek C, Becker JB. The development of a preference for cocaine over food identifies individual rats with addiction-like behaviors. PLoS One 2013; 8 (11): e79465.

10. Friedewald WT, Levy RI, Fredrickson DS. Estimation of the concentration of low-density lipoprotein cholesterol in plasma, without use of the preparative ultracentrifuge. Clin Chem 1972; 18 (6): 499-502.

11. Acevedo M, Krämer V, Tagle R, Corbalán R, Arnaíz P, Berríos X, et al. [Total/HDL cholesterol ratio and non HDL cholesterol as predictors for increased intima media thickness]. Rev Med Chile 2012; 140 (8): 969-76.

12. Verdejo-García A, Pérez-García M, Sánchez-Barrera M, Rodríguez-Fernández A, Gómez-Río M. [Neuroimaging and drug addiction: neuroanatomical correlates of cocaine, opiates, cannabis and ecstasy abuse]. Rev Neurol 2007; 44 (7): 432-9.

13. Spronk DB, van Wel JH, Ramaekers JG, Verkes RJ. Characterizing the cognitive effects of cocaine: a comprehensive review. Neurosci Biobehav Rev 2013; 37 (8): 1838-59.

14. Saez CG, Olivares P, Pallavicini J, Panes O, Moreno N, 
Massardo $\mathrm{T}$, et al. Increased number of circulating endothelial cells and plasma markers of endothelial damage in chronic cocaine users. Thromb Res 2011; 128 (4): e18-23.

15. Pereira J, Sáez CG, Pallavicini J, Panes O, Pereira-Flores $\mathrm{K}$, Cabreras MJ, et al. Platelet activation in chronic cocaine users: effect of short term abstinence. Platelets 2011; 22 (8): 596-601.

16. Massardo T, Quintana J, Jaimovich R, Sáez C, Pallavicini J, Véliz J, et al. Association between endothelial dysfunction markers and regional cerebral blood flow (rCBF) changes in cocaine dependent patients under recent abstinence. Eur J Nucl Med Mol Imaging 2013; 40 (Suppl 2): 340-1.

17. Ramos-Aliaga R, Placencia M. Effect of cocaine intake on the development of fatty liver in rats fed a low-protein diet. Arch Latinoam Nutr 1987; 37 (2): 282-94.

18. Odeleye OE, López MC, Smith BT, Eskelson CD, Watson RR. Cocaine hepatotoxicity during protein undernutrition of retrovirally infected mice. Can J Physiol Pharmacol 1992; 70 (3): 338-43.

19. Vitcheva V. Cocaine toxicity and hepatic oxidative stress. Curr Med Chem 2012; 19 (33): 5677-82.

20. Visalli T, Turkall R, Abdel-Rahman MS. Influence of gender on cocaine hepatotoxicity in CF-1 mice. Int J Toxicol 2005; 24 (1): 43-50.

21. Evans SM, Foltin RW. Does the response to cocaine differ as a function of sex or hormonal status in human and non-human primates? Horm Behav 2010; 58 (1): 13-21.

22. Anker JJ, Carroll ME. Females are more vulnerable to drug abuse than males: evidence from preclinical studies and the role of ovarian hormones. Curr Top Behav Neurosci 2011; 8: 73-96.

23. Ersche KD, Stochl J, Woodward JM, Fletcher PC. The skinny on cocaine: Insights into eating behavior and body weight in cocaine-dependent men. Appetite 2013; 71: 75-80.

24. Douglass J, McKinzie AA, Couceyro P. PCR differential display identifies a rat brain mRNA that is transcriptionally regulated by cocaine and amphetamine.
J Neurosci 1995; 15 (3 Pt 2): 2471-81.

25. Parker JA, Bloom SR. Hypothalamic neuropeptides and the regulation of appetite. Neuropharmacology 2012; 63 (1): 18-30.

26. De Luis DA, Armentia A, Muñoz PL, Dueñas-Laita A, Martín B, De la Fuente B, et al. [Dietary intakes in a group of marihuana smoking patients]. Nutr Hosp 2010; 25 (4): 688-91.

27. Rott D, Langleben DD, Elman I. Cocaine decreases plasma insulin concentrations in non-diabetic subjects: a randomized double-blind study. Diabet Med 2008; 25 (4): 510-1.

28. Ersche KD, Hagan CC, Smith DG, Abbott S, Jones PS, Apergis-Schoute AM, et al. Aberrant Disgust Responses and Immune Reactivity in Cocaine-Dependent Men. Biol Psychiatry 2014; 75 (2): 140-7.

29. Fox HC, D'Sa C, Kimmerling A, Siedlarz KM, Tuit $\mathrm{KL}$, Stowe R, et al. Immune system inflammation in cocaine dependent individuals: implications for medications development. Human Psychopharmacol 2012; 27 (2): 156-66.

30. Shattil SJ, Anaya-Galindo R, Bennett J, Colman RW, Cooper RA. Platelet hypersensitivity induced by cholesterol incorporation. J Clin Invest 1975; 55 (3): 636-43.

31. Shattil SJ, Bennett JS, Colman RW, Cooper RA. Abnormalities of cholesterol-phospholipid composition in platelets and low-density lipoproteins of human hyperbetalipoproteinemia. J Lab Clin Med 1977; 89 (2): 341-53.

32. Puccetti L, Santilli F, Pasqui AL, Lattanzio S, Liani $\mathrm{R}$, Ciani F, et al. Effects of atorvastatin and rosuvastatin on thromboxane-dependent platelet activation and oxidative stress in hypercholesterolemia. Atherosclerosis 2011; 214 (1): 122-8.

33. Walker J, Winhusen T, Storkson JM, Lewis D, Pariza $\mathrm{MW}$, Somoza E, et al. Total antioxidant capacity is significantly lower in cocaine-dependent and methamphetamine-dependent patients relative to normal controls: results from a preliminary study. Hum Psychopharmacol 2014; 29 (6): 537-43. 